03

\title{
Интенсификация ламинарного течения в узком микроканале с однорядными наклоненными овально-траншейными лунками
}

\author{
() С.А. Исаев ${ }^{1,2}$, П.А. Баранов ${ }^{2}$, А.И. Леонтьев ${ }^{3}$, И.А. Попов ${ }^{1}$ \\ ${ }^{1}$ Казанский национальный исследовательский технический университет \\ им. А.Н. Туполева - Казанский авиационный институт, Казань, Россия \\ ${ }^{2}$ Санкт-Петербургский государственный университет гражданской \\ авиации, Санкт-Петербург, Россия \\ ${ }^{3}$ Московский государственный технический университет \\ им. Н.Э. Баумана, Москва, Россия \\ E-mail: isaev3612@yandex.ru
}

Поступило в Редакцию 3 октября 2017 г.

С помощью разработанных многоблочных вычислительных технологий при $\mathrm{Re}=10^{3}$ проведены расчеты полностью развитого ламинарного течения воздуха в плоскопараллельном канале шириной 6 и высотой 1 с нанесенными на стенку однорядными наклоненными овально-траншейными лунками. Рассматривается периодическая секция канала длиной 4 с одной траншейной лункой длиной 4.5 , шириной 1 , углом наклона к потоку $45^{\circ}$ при изменении глубины от 0 до 0.375. Обнаружена интенсификация ламинарного течения в ядре потока в облуненном канале при глубине траншейных лунок свыше 0.25 с увеличением максимальной скорости более чем в 1.5 раза по сравнению с максимальной скоростью потока в гладком канале.

DOI: 10.21883/PJTF.2018.09.46068.17066

Интенсификация ламинарного течения в каналах и трубах представляет собой сложную проблему. Для ускорения потока требуется увеличивать перепад полного давления, что не всегда возможно. Проблема важна для повышения эффективности теплоэнергетического оборудования. Она связана с интенсификацией теплообмена с помощью поверхностной дискретной шероховатости, включая упорядоченные лунки, размещение в трубах скрученных лент, изменение формы проточного тракта за счет создания периодических конфузорно- 
диффузорных секций [1-3]. В последнее десятилетие усилился интерес к микроканалам [4-6], в которых увеличение теплоотдачи вызывается расположением на стенке однорядного пакета сферических лунок. Однако сферические лунки не являются эффективными интенсификаторами, хотя при низких числах Рейнольдса Re отмечается снижение гидравлических потерь в облуненном канале (dimpled channel) по сравнению с таковыми для гладкого плоскопараллельного аналога.

В 2003 г. [7] были предложены овальные лунки, расположенные с наклоном к набегающему потоку под углом $\theta$, с длиной цилиндрической вставки $L$ между двумя разнесенными половинами сферической лунки диаметром $d$ и глубиной $\Delta$. В [8] при турбулентном обтекании пластины с овальной лункой показано, что такие лунки являются более эффективными поверхностными вихрегенераторами, чем их сферические аналоги, а оптимальный угол наклона овальной лунки к потоку составляет $45^{\circ}$. Однорядные пакеты овальных лунок с $L=0.8$, шириной 1 , и глубиной 0.2 , нанесенные на стенку плоскопараллельного канала высотой 0.44 и шириной 2.5 с шагом 1.8 , рассматриваются в [9] при ламинарном режиме течения с Re, изменяющимся от 100 до 2500 (число Рейнольдса Re определено по ширине лунки и среднемассовой скорости). Однако овальные лунки умеренной длины не являются рациональными по гидравлическим потерям. Как показано в [10], при уменьшении ширины овальной лунки и сохранении постоянными площади пятна и глубины лунки гидравлические потери в облуненном узком канале при турбулентном течении достигают максимума, после чего снижаются до уровня, практически соответствующего гидравлическим потерям в канале с эквивалентной по площади пятна сферической лункой (в этом случае длина овально-траншейной лунки в 6 раз превышает ширину).

В настоящей работе решается задача об интенсификации ламинарного течения воздуха при фиксированном числе Рейнольдса $\operatorname{Re}=10^{3}$ в узком канале прямоугольного поперечного сечения шириной 6 и высотой 1 с нанесенным на нижнюю стенку пакетом однорядных овально-траншейных лунок с шагом 4 и наклоном $45^{\circ}$ к продольной оси канала. Ширина лунок 1 , длина 4.5 , радиус скругления кромок 0.025 . Глубина лунки $\Delta$ изменяется в пределах от 0 до 0.375 . Вводится система декартовых координат $x, y, z$ с центром в начале расчетного модуля, ось $x$ которой ориентируется вдоль, а ось $z-$ поперек канала. Все размеры отнесены к высоте канала. Декартовы составляющие скорости

Письма в ЖТФ, 2018, том 44, вып. 9 
Влияние расчетной сетки на экстремальные величины локальных декартовых составляющих скорости и на коэффициент гидравлических потерь $\xi$

\begin{tabular}{r|c|c|c|c|c|c|c}
\hline$N \cdot 10^{-3}$ & $u_{\max }$ & $u_{\min }$ & $v_{\max }$ & $v_{\min }$ & $w_{\max }$ & $w_{\min }$ & $\xi \cdot 10^{2}$ \\
\hline 650 & -0.136 & 2.139 & -0.201 & 0.165 & -0.572 & 0.116 & 3.273 \\
1150 & -0.154 & 2.130 & -0.203 & 0.168 & -0.560 & 0.118 & 3.250 \\
2500 & -0.155 & 2.135 & -0.202 & 0.167 & -0.558 & 0.118 & 3.244
\end{tabular}

$u, v, w$ и число $\operatorname{Re}$ определяются по среднемассовой скорости потока в канале.

Система стационарных уравнений Навье-Стокса для несжимаемой среды решается конечно-объемным факторизованным методом с помощью многоблочных вычислительных технологий, основанных на пересекающихся разномасштабных сетках [11]. На проточных границах выделенного модуля с лункой ставятся периодические граничные условия, а на стенках - условия прилипания. При решении задачи применяется процедура коррекции давления [8,11].

На рис. 1-3 и в таблице представлены некоторые из полученных результатов.

Методические расчеты выполнены на многоблочных сетках различного типа и количества ячеек $N$. Простейшая и наиболее грубая сетка, содержащая порядка 650 тысяч ячеек (рис. 1,a), состоит из двух фрагментов: прямоугольной канальной сетки с равномерными шагами $(0.1)$ вдоль и поперек на центральном участке $(4 \times 4)$ периодического модуля, а также криволинейной пристеночной сетки, согласованной с поверхностью лунки высотой 0.2, с измельчением продольного и поперечного шага (0.05). Шаги у стенок равны 0.0001 . Уточненная сетка, содержащая порядка 1 миллиона 150 тысяч ячеек, включает дополнительные криволинейные сетки в окрестности кромок и внутри лунки. И наконец, наиболее измельченная сетка с количеством ячеек в 2 миллиона 500 тысяч характеризуется двукратным уменьшением продольных и поперечных максимальных шагов в канальном и пристеночном фрагментах многоблочной сетки.

В таблице сравниваются экстремальные величины параметров течения и гидравлические потери в периодическом модуле, рассчитанные на разных многоблочных сетках. Как следует из таблицы, по мере

Письма в ЖТФ, 2018, том 44, вып. 9 

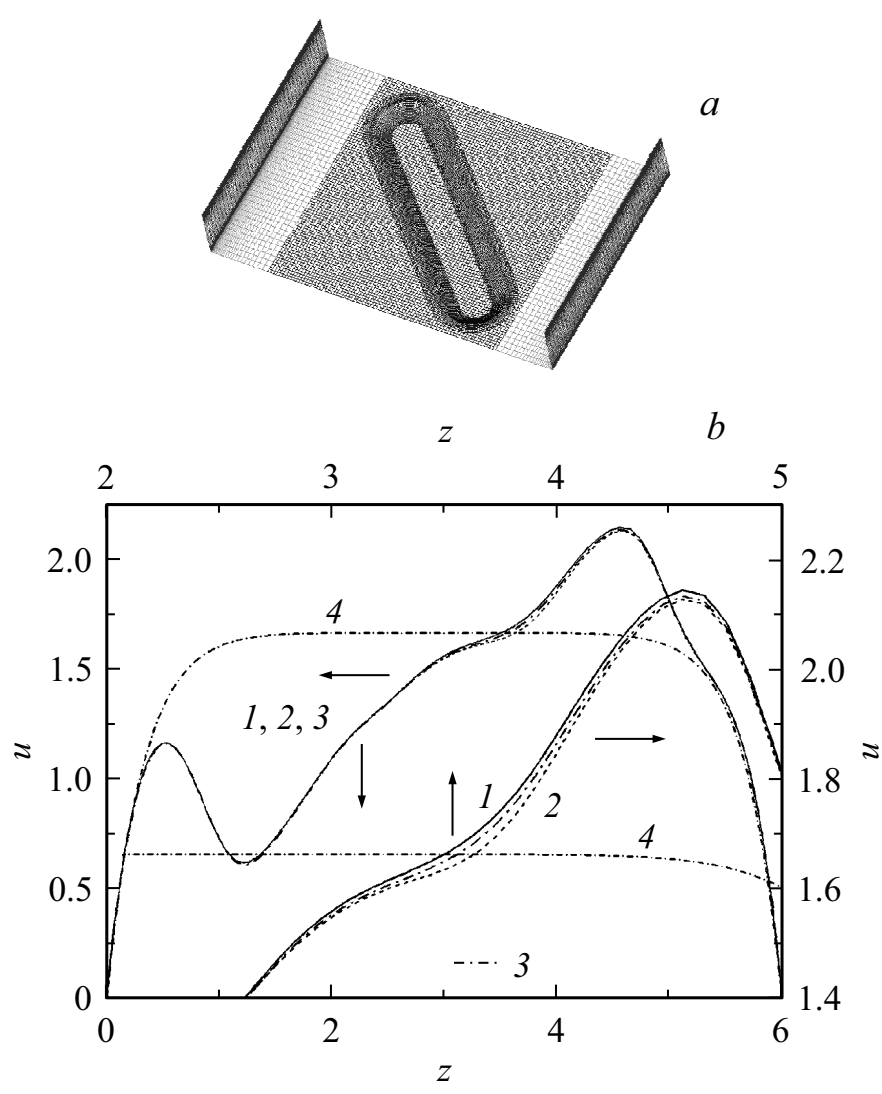

Рис. 1. Многоблочные сетки в периодическом модуле с овально-траншейной лункой глубиной $0.25(a)$ и сравнение (в двух масштабах, включая укрупненный) профилей продольной составляющей скорости $u(z)$ в сечениях $x=1(1)$, $2(2), 3(3)$ для фиксированных $y=0.35$ и $\operatorname{Re}=10^{3}(b)$. Кривые 4 соответствуют профилю $u(z)$ плоскопараллельного канала при $y=0.5$.

измельчения сетки растет прогнозируемая интенсификация возвратного течения, хотя максимальная (по абсолютной величине) скорость вторичного течения монотонно снижается, как и коэффициент гидравлических потерь.

Письма в ЖТФ, 2018, том 44, вып. 9 


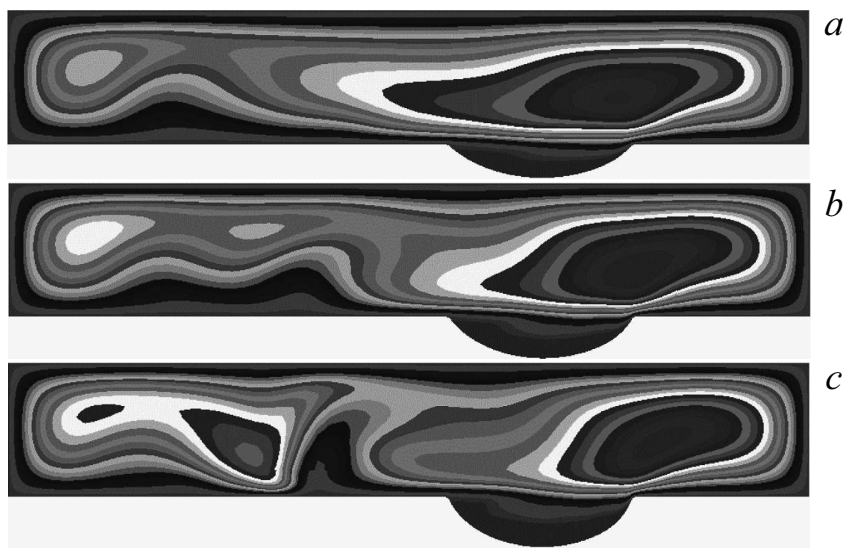

Рис. 2. Картины поперечных полей изолиний продольной скорости $u$, построенных с шагом 0.1 от 0 до 2.1, при глубинах $\Delta=0.25(a), 0.3125(b)$ и 0.375 (c) в срединном поперечном сечении периодического модуля плоскопараллельного канала $(x=2)$.

Как видно из рис. $1, b$, поле продольной скорости в ядре потока в канале с наклоненной овально-траншейной лункой оказывается сильно неоднородным и практически не меняется с увеличением расстояния от входного сечения периодического модуля. В правой половине канала с лункой возникает зона повышенной продольной составляющей скорости, в 1.5 раза превосходящей максимальную скорость в плоскопараллельном канале. Таким образом, открыт эффект интенсификации ламинарного потока в узком канале с периодическими овально-траншейными лунками умеренной глубины (25\% от ширины) с ускорением течения в 1.5 раза по сравнению с гладким каналом (2.15 от среднемассовой скорости). В левой половине канала образуются локальный максимум $u$, примерно в 1.3 раза уступающий по величине максимальной скорости в гладком плоскопараллельном канале, и локальный минимум $u$, равный $60 \%$ от среднемассовой скорости.

Трансформация полей продольной скорости в срединном поперечном сечении облуненного канала с ростом глубины $\Delta$ овальнотраншейной лунки на рис. 2 демонстрирует интенсификацию ламинарного течения в формирующихся зонах локальных максимумов скорости,

Письма в ЖТФ, 2018, том 44, вып. 9 


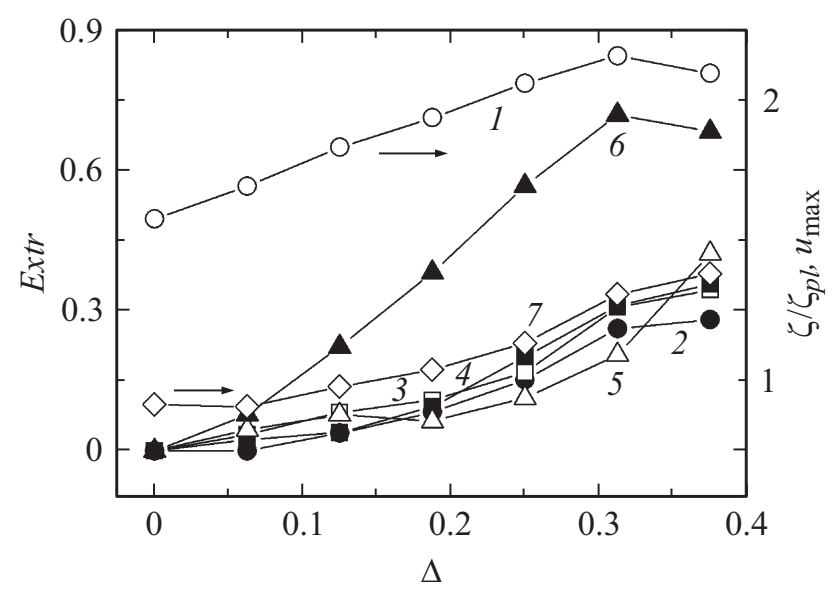

Рис. 3. Зависимости экстремальных величин параметров потока Extr и гидравлических потерь от глубины лунки $\Delta .1-u_{\max }, 2-\left(-u_{\min }\right), 3-v_{\max }$, $4-\left(-v_{\min }\right), 5-w_{\max }, 6-\left(-w_{\min }\right), 7-\xi / \xi_{p l}$.

а также усиление степени неоднородности в ядре канального потока, в особенности при $\Delta$ не менее 0.25 (рис. 2,a). Так, при $\Delta=0.3125$ возникает еще одна область повышенных скоростей в левой половине канала (рис. $2, b$ ), а при $\Delta=0.375$ она приближается к нижней стенке и рядом с ней возникает зона пониженных скоростей (рис. 2,c).

Поведение экстремальных параметров потока Extr в облуненном канале с ростом $\Delta$, показанное на рис. 3 (кривые $1-6$ ), согласуется с зависимостью $\xi / \xi_{p l}(\Delta)$ для коэффициента гидравлических потерь облуненного канала $\xi$, отнесенного к коэффициенту гидравлических потерь в гладком канале $\xi_{p l}$ (кривая 7). Увеличение $\Delta$ сопровождается интенсификацией ламинарного течения в канале, в том числе вторичного и возвратного течения, а также приводит к монотонному росту гидравлических потерь. Максимальная величина продольной составляющей скорости течения растет линейно при изменении глубины лунки от 0 до 0.3125. Важно отметить, что наибольшая скорость вторичного течения достигает $70 \%$ от среднемассовой скорости, а продольная составляющая скорости -2.25 . Максимальные относительные гидравлические потери в облуненном канале при $\Delta=0.375$ составляют 1.44 .

Письма в ЖТФ, 2018, том 44, вып. 9 
Интересно провести аналогию между течением в турбомашинах $[12,13]$ и в рассматриваемом плоскопараллельном канале с нанесенными на стенку однорядными овально-траншейными лунками с наклоном по отношению к потоку под углом $45^{\circ}$. Эффект ускорения потока в таком плоскопараллельном канале объясняется воздействием на ламинарное течение решетки утопленных в стенку профилей овальных удлиненных траншей с глубиной не менее 0.25 . Увеличение в 1.5 раза максимальной скорости в ядре потока в облуненном канале по сравнению с максимальной скоростью в гладком канале сочетается с формированием сильно неоднородного течения.

Таким образом, открытое явление интенсификации ламинарного потока в узком плоскопараллельном облуненном канале при $\operatorname{Re}=10^{3}$ связано с ростом в 1.5 раза максимальной скорости полностью развитого течения при нанесении на стенку однорядных овально-траншейных лунок, наклоненных под углом $45^{\circ}$ к продольной оси канала. Лунки имеют длину 4.5, ширину 1 и глубину не менее 0.25 в долях высоты канала.

Работа выполнена при государственной поддержке научных исследований, проводимых под руководством ведущих ученых в российских вузах (ведущий ученый - С.А. Исаев, КНИТУ - КАИ, Казань) по гранту Правительства России №14.Z50.31.0003.

\section{Список литературы}

[1] Назмеев Ю.Г. Теплообмен при ламинарном течении жидкости в дискретношероховатых каналах. М.: Энергоатомиздат, 1998. $371 \mathrm{c.}$

[2] Дзюбенко Б.В., Кузма-Кичта Ю.А., Леонтьев А.И., Федик И.И., Холпанов Л.П. Интенсификация тепло- и массообмена на макро-, микро- и наномасштабах. М.: ЦНИИАТОМИНФОРМ, 2008. $532 \mathrm{c}$.

[3] Вихревые технологии для энергетики / Под общ. ред. акад. А.И. Леонтьева. М.: Изд. дом МЭИ, 2016. 350 с.

[4] Wei X.J., Joshi Y.K., Ligrani P.M. // J. Electron. Package. 2007. V. 129. N 1. P. 63-70.

[5] Lee J., Lee K.-S. // Int. J. Heat Mass Transfer. 2013. V. 64. N 5. P. 444-451.

[6] Xu M., Lu H., Gong L., Chai J.C., Duan X. // Int. Commun. Heat Mass Transfer. 2016. V. 76. P. $348-357$.

[7] Исаев С.А., Леонтьев А.И., Митяков А.В., Пышный И.А. // Инж.-физ. журн. 2003. T. 76. № 2. C. 31-34.

Письма в ЖТФ, 2018, том 44, вып. 9 
[8] Быстров Ю.А., Исаев С.А., Кудрявщев Н.А., Леонтьев А.И. Численное моделирование вихревой интенсификации теплообмена в пакетах труб. СПб.: Судостроение, 2005. 398 с.

[9] Исаев С.А., Леонтьев А.И., Корнев Н.В., Хассель Э., Чудновский Я.П. // Теплофизика высоких температур. 2015. Т. 53. № 3. С. 390-402.

[10] Исаев С.А., Леонтьев А.И., Гульцова М.Е., Попов Ю.А. // Письма в ЖТФ. 2015. T. 41. B. 12. C. 89-96.

[11] Исаев С.А., Баранов П.А., Усачов А.Е. Многоблочные вычислительные технологии в пакете VP2/3 по аэротермодинамике. Саарбрюккен: LAP LAMBERT Academic Publ., 2013. $316 \mathrm{c}$.

[12] Гастелоу Джс. Аэродинамика решеток турбомашин. М.: Мир, 1987. 392 с.

[13] Степанов Г.Ю. Гидродинамика решеток турбомашин. М.: Физматгиз, 1962. $512 \mathrm{c}$. 